Jpn. J. Genet. (1995) 70, pp. 687-692

\title{
NOTES
}

\section{Molecular clock for dating of divergence between animal phyla}

\author{
Naoyuki Iwabe, Kei-ichi Kuma, Naruo NikoH \\ and Takashi MiYata* \\ Department of Biophysics, Faculty of Science, Kyoto University, \\ Kyoto 606-01 Japan
}

(Received 3 August 1995)

\begin{abstract}
To estimate approximate times of divergence of animal phyla lacking fossil data, it is important to find a molecule that evolves with an approximately constant rate over a wide evolutionary distance covering the whole animal phyla. For this purpose, the evolutionary rate constancy has been examined for 20 proteins. It was found that four proteins, particularly the aldolase $\mathrm{C}$, involved in the glycolitic pathway, had evolved with rates that are approximately constant not only among different classes of vertebrates, but also between vertebrates and arthropods. The evolutionary rate $\left(=0.26 \times 10^{-9} /\right.$ site/year $)$ of the aldolase $\mathrm{C}$ is likely to have remained essentially unchanged even between animals and plants.
\end{abstract}

It is generally thought that molecules accumulate amino acid substitutions at approximately constant rates with respect to time during vertebrate evolution, when the sequences are compared between different classes of vertebrates (Zuckerkandl and Pauling, 1965; Wilson et al., 1977; Dickerson and Geis, 1983; Kimura, 1983; Nei 1987). The clock like behavior of molecules, however, is not always observed, when compared between different orders of mammals, and the rates of evolution often differ for different taxonomic groups (Britten, 1986). The molecular clock is applicable only to dating of divergence between distantly related groups (e.g. Nei, 1987). Furthermore there is no evidence supporting the clock like behavior of molecules other than vertebrates, except for cytochrome $c$ (Dickerson, 1971). To estimate the approximate time of divergence between animal phyla, it is important to find an appropriate molecule which has evolved with an approximately constant rate over a wide evolutionary distance covering the whole animal phyla. We report here that aldolase has evolved with a rate that is approximately constant between vertebrates and arthropods and possibly even between animals and fungi or plants and thus is useful for dating of divergence between animal phyla.

From a phylogenetic analysis of 26 different gene families, we previously

* Corresponding author. 
showed that, after the separation from arthropods, chordates had undergone extensive gene duplications and rapid amino acid substitutions on the ancestral lineage before the divergence of fishes and tetrapods (Iwabe et al. 1996). The aldolase family, however, showed an exceptionally low genetic diversity. This result suggests that in these molecules except for aldolase, the constant rate of evolution is recognized only on the lineages of jawed vertebrates. To confirm this, the evolutionary rate constancy was examined for 20 different proteins, including proteins specific to multicellular organisms, enzymes involved in the glycolytic pathway, the elongation factor $1 \alpha$ and the cytochrome $c$.

Amino acid sequences were compared between different mammalian orders, between mammals and birds or reptiles, between amniotes and amphibians, between fishes and tetrapods, between gnathostomes and lamprey, between chordates and arthropods, and between animals and fungi or plants, and for each pair of groups the average value $\mathrm{K}_{\mathrm{aa}}$ of sequence differences between species compared was calculated, from which the average number $\mathrm{k}_{\mathrm{aa}}$ of amino acid substitutions per site was obtained by correcting multiple substitutions by the method of Jukes and Cantor (1969). All the sequence data were taken from GenBank release 87.0 and PIR database release 43.0, except for chicken aldolase C (Ono et al., 1990) and lamprey non-muscle-type aldolase (Zhang et al., 1995). The kaa values were plotted against the divergence times T's of taxonomic groups compared. The following divergence times were assumed; 75 million years (myrs) for the divergence of different mammalian orders (Dayhoff, 1978), 300 myrs for mammals - birds/reptiles divergence (Dayhoff, 1978), 350 myrs for amniotes - amphibians divergence (Dayhoff, 1978), 400 myrs for tetrapods - fishes divergence (Dayhoff, 1978), 500 myrs for gnathostomes-lamprey divergence (Dickerson, 1971), 700 myrs for chordates - arthropods divergence (Dayhoff, 1978, Margulis and Schwartz, 1988). To exclude a possibility of paralogous comparison, a phylogenetic tree was inferred by the neighbor - joining method (Saitou and Nei, 1987) for each protein by including many sequences available from databases.

Fig. 1 shows the relationships between the number $k_{a a}$ of amino acid substitutions per site and the time $\mathrm{T}$ of divergence between species compared. Proteins shown in Fig. 1a-1e are mostly found in animals and those shown in Fig. 1f-1j are ubiquitous and are found in all organisms. The evolutionary rates of the animalspecific proteins shown in Fig. 1a-1e are approximately constant, when compared between vertebrate groups. However, the point corresponding to vertebratesarthropods comparison evidently places above the regression lines. Seven more proteins specific to animals were also examined, and essentially identical results were obtained. In contrast, four proteins, the aldolase $\mathrm{C}$, the phosphoglycerate kinase (PGK1), the triose-phosphate isomerase (TPI), and the enolase $\alpha$, involved in the glycolitic pathway, show different patterns: In each molecule, an approximate linear dependence of $\mathrm{k}_{\mathrm{aa}}$ on $\mathrm{T}$ was found, even if the point corresponding to vertebrates-arthropods comparison was included (Fig. 1f-1i). Two more enzymes 
involved in the glycolitic pathway were also examined. The alchol dehydrogenase did not show a good linearity, possibly due to paralogy of vertebrate and arthropod proteins. Shih et al. (1986) proposed that the glyceraldehyde dehydrogenase (GAPDH) evolves with an approximately constant rate over a wide evolutionary distance, and thus the GAPDH clock is useful for inferring the divergence times of diverse groups of organisms. However, since only three points corresponding to human-rat, mammals-birds and vertebrates-arthropods pairs are available at present, it is not certain whether this enzyme behaves like a clock or not.

Each of proteins shown in Fig. 1a-1e belongs to a large protein family, and the family members are often expressed tissue specifically. Recently we showed that a group of family members carrying virtually identical function to one another, but differing in tissue distribution (tissue-specific isoforms) had undergone extensive gene duplications and large amounts of amino acid substitutions during the early evolution of chordates before the separation of fishes and tetrapods (Iwabe et al. 1996). A similar genetic diversification may have occurred on the arthropod lineage. The extensive genetic variations in the early evolution of chordates may be responsible for the large amount of amino acid substitutions between vertebrates and arthropods. On the other hand, in the four enzymes involved in the glycolitic pathway (Fig. 1f-1i), only two or three tissue-specific isoforms exist in vertebrates. It should be noted that house keeping molecules do not always behave like a clock over a wide evolutionary distance, as demonstrated by the elongation factor $1 \alpha$ (Fig. 1j), although in this case, a possibility of paralogous comparison can not be excluded.

In the case of the aldolase $\mathrm{C}$, the linear dependence of $\mathrm{k}_{\mathrm{aa}}$ on $\mathrm{T}$ is particularly excellent, and the date of divergence of animals and plants has been estimated to be about $1150 \pm 110$ myrs from the aldolase clock, which is close to a generally accepted date of 1000 myrs for the divergence of animals and fungi (Dickerson 1971; Dayhoff 1978). Note that, because animals are more closely related to fungi than to plants (Wainright 1993; Hasegawa et al. 1993; Nikoh et al. 1994), the estimate of time earlier than 1000 myrs may be reasonable. Thus the aldolase $\mathrm{C}$ clock may be useful for inferring the time of divergence between animal phyla. Vertebrates have three distinct types of aldolases, aldolase A, B, and C, which differ to one another in tissue distribution (Zhang et al., 1995). These isoforms diverged on vertebrate lineage, independently from arthropods, and the two phyla share only a single common precursor. The evolutionary rate of the aldolase $\mathrm{B}$ is evidently higher than that of the aldolase C (Zhang et al., 1995) and is not constant over a wide evolutionary time span. The molecular clock analysis of the aldolase $\mathrm{A}$ is not possible at present, because the sequence data are available only for mammals.

The molecular clock of cytochrome $\mathrm{c}$ was reexamined based on a new data set. The linear relationship between $\mathrm{k}_{\mathrm{aa}}$ and $\mathrm{T}$ was observed within the limit of the 
a)

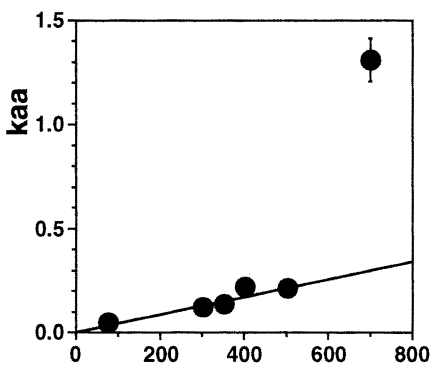

b)

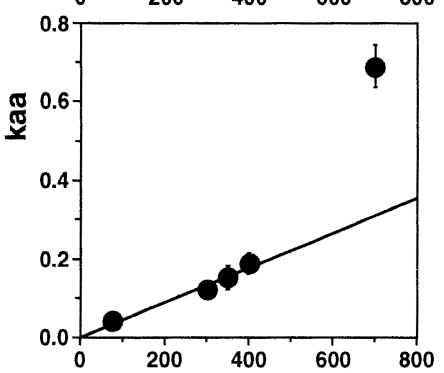

c)

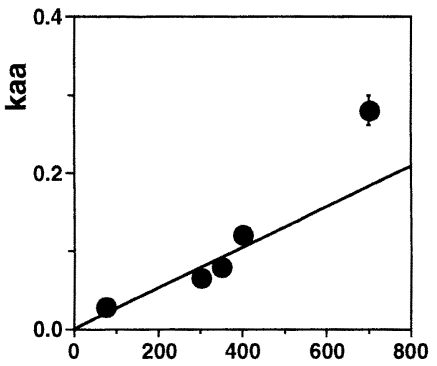

d)

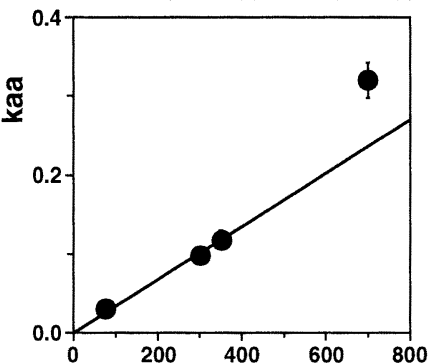

e)

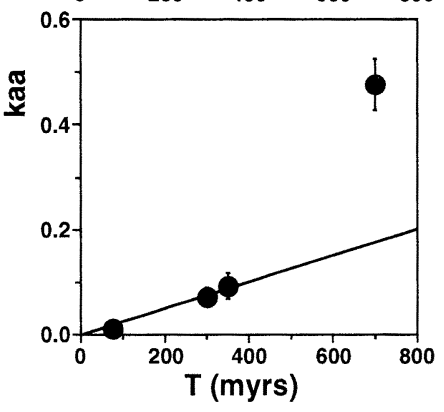

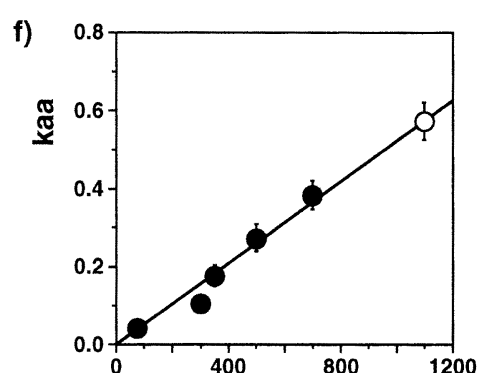
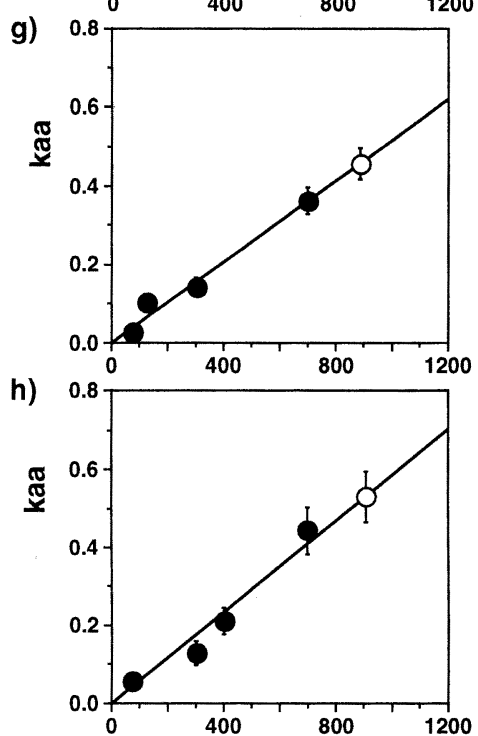

i)
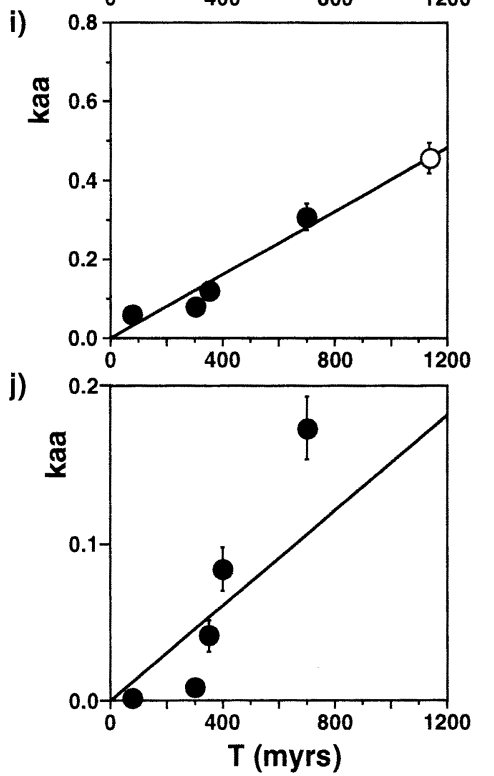
standard error $\sigma$. However, the $\sigma$ value of each point is considerably large due to the small number of sites available for comparison, and an inferred phylogenetic tree includes several unrealistic tree topologies among taxonomic groups (data not shown). The clock like behavior of aldolase $\mathrm{C}$ over a wide evolutionary distance would also allows ones to infer an accurate phylogenetic tree among animal phyla: The evolutionary rate of 18SrRNAs of Radiata differs widely from that of Bilateria (Wainright 1993). Since a wide difference of evolutionary rates between different lineages often leads to an erroneous tree, reexamination of the 18SrRNA tree by protein molecules like the aldolase $\mathrm{C}$ may be required.

This work was supported in part by grants from the Ministry of Education, Science, Sports and Culture of Japan.

\section{REFERENCES}

Britten, R. J. (1986). Rates of DNA sequence evolution differ between taxonomic groups. Science 231, 1393-1398.

Dayhoff, M. O. (1978). Survey of new data and computer methods of analysis. In: Atlas of Protein Sequence and Structure (ed.: M. O. Dayhoff), pp. 1-8. National Biomedical Research Founda-

Fig. 1. The plot of $\mathrm{k}_{\mathrm{aa}}$, the number of amino acid substitutions per residue, versus $\mathrm{T}$, the time of divergence of different species. a), rhodopsin. The number $n_{\mathrm{aa}}$ of amino acid sites compared is 272 and the rate $\mathrm{v}_{\mathrm{aa}}$ of evolution estimated from the regression line is $0.22 \times 10^{-9} / \mathrm{site} / \mathrm{year}$. b), nicotinic actylchorine receptor $\alpha$ subunit. $\left.\mathrm{n}_{\mathrm{aa}}=358 ; \mathrm{v}_{\mathrm{aa}}=0.22 \times 10^{-9} . \quad \mathrm{c}\right), \mathrm{Na}^{+}-\mathrm{K}^{+}$ATPase $(\alpha-1) . \quad \mathrm{n}_{\mathrm{aa}}=976$; vaa $=0.13 \times 10^{-9}$. d), sarcoplasmic/endoplasmic reticulum $\mathrm{Ca}^{2+}$ ATPase (SERCA1). $\mathrm{n}_{\mathrm{aa}}=990$; $\mathrm{v}_{\mathrm{aa}}=0.17 \times 10^{-9}$. e), muscarinic acetylchorine receptor $(\mathrm{m} 4) . \quad \mathrm{n}_{\mathrm{aa}}=252 ; \mathrm{v}_{\mathrm{aa}}=0.12 \times 10^{-9}$. f), aldolase C. $\left.\mathrm{n}_{\mathrm{aa}}=353 ; \quad \mathrm{v}_{\mathrm{aa}}=0.26 \times 10^{-9} . \mathrm{g}\right)$, phosphoglycerate kinase (PGK1). $\mathrm{n}_{\mathrm{aa}}=411$; $\mathrm{v}_{\mathrm{aa}}=0.26 \times 10^{-9} . \mathrm{h}$ ), triose phosphate isomerase. $\mathrm{n}_{\mathrm{aa}}=245 ; \mathrm{v}_{\mathrm{aa}}=0.29 \times 10^{-9}$. i), enolase $\alpha$. $\mathrm{n}_{\mathrm{aa}}=431 ; \mathrm{v}_{\mathrm{aa}}=0.20 \times 10^{-9} . \mathrm{j}$ ), elongation factor $1 \alpha . \mathrm{n}_{\mathrm{aa}}=461 ; \mathrm{v}_{\mathrm{aa}}=0.07 \times 10^{-9}$. The regression lines were calculated for the points corresponding to the comparisons between different groups of vertebrates for a) - e) and $j$ ) and those between different groups of vertebrates and between vertebrates and arthropods for $\mathrm{f}$ ) - i). Open circle, the divergence time $\mathrm{T}$ was estimated by assuming the constant rate of evolution. The standard error of each point was calculated as $\sqrt{\mathrm{K}_{\mathrm{aa}} /\left[\left(1-\mathrm{K}_{\mathrm{aa}}\right) \mathrm{n}_{\mathrm{aa}}\right]}$ (Kimura 1983), whe-e $\mathrm{K}_{\mathrm{aa}}$ is the average value of sequence differences between species compared. Accession numbers of sequences used: a), human, K02281; mouse, M55171, M36699; chicken, D00702; Rana, S49004; Carassius, L11863; Lampetra, M63632; Drosophila (opsin Rh1), K02315. b), human, Y00762, X02508; mouse, X03986; chicken, X07338; Xenopus, X07067; Torpedo, J00963; Drosophila ( $\alpha$-like), X07194. c), human, X04297, D00099; rat, M14511, X05882, D00188, D10359; chicken, J03230; Xenopus, U10108; Catostomus, X58629; Drosophila, X14476. d), rat, M99223; rabbit, M12898; chicken, M26064; Rana, X63009; Drosophila, M62892. e), human, X15265; mouse, X63473; chicken, J05218; Xenopus, X65865; Drosophila, M23412. f), human, X05196; rat, X06984; chicken, Ono et al. 1990; Xenopus, S73606; Entosphenus (non-muscle-type), Zhang et al. 1995; Drosophila, M98351; Arabidopsis, X53058. g), human, M11968; mouse, M15668; tammar wallaby, X64296; chicken, L37101; Drosophila, Z14029; Saccharomyces, X59720. h), human, M10036; mouse, X53333; chicken, M11941; coelacanth, A01167 (PIR); Drosophila, X57576; Arabidopsis, U02949. i), human, M14328; mouse, X52379; duck, M20749; Xenopus, Y00718; Drosophila, X17034; Saccharomyces, J01322. j), human, X03558, J04617; rat, X61043, X63561; chicken, L00677; Xenopus, X55324, M25697; Brachydanio, L23807, X77689; Drosophila (F1), M11744, X06869. 
tion, Washington, DC.

Dickerson, R. E. (1971). The structure of cytochrome $c$ and the rates of molecular evolution. J. Mol. Evol. 1, 26-45.

Dickerson, R. E. and Geis, I. (1983). Hemoglobin. Benjamin/Cummings, Menlo Park, CA.

Hasegawa, M., Hashimoto, T., Adachi, J., Iwabe, N. and Miyata, T. (1993). Early branching in the evolution of eukaryotes: ancient divergence of Entamoeba that lacks mitochondria revealed by protein sequence data. J. Mol. Evol. 36, 380-388.

Iwabe, N., Kuma, K. and Miyata, K. (1996). Evolution of gene families and relationship with organismal evolution: Rapid divergence of tissue-specific genes in the early evolution of chordates. Mol. Biol. Evol. in press.

Jukes, T. H. and Cantor, C. R. (1969). Evolution of protein molecules. In: Mammalian Protein Metabolism III (ed: H. N. Munro), pp. 21-132. Academic Press, New York.

Kimura, M. (1983). The Neutral Theory of Molecular Evolution. Cambridge University Press, Cambridge.

Margulis, L. and Schwartz, K. V. (1988). Five kingdoms: An illustrated guide to the phyla of life on earth. 2nd ed. Freeman and Co., New York.

Nei, M. (1987). Molecular Evolutionary Genetics. Columbia University Press, New York.

Nikoh, N., Hayase, N., Iwabe, N., Kuma, K. and Miyata, T. (1994). Phylogenetic relationship of the kingdoms Animalia, Plantae, and Fungi inferred from twenty three different protein species. Mol. Biol. Evol. 11, 762-768.

Ono, K., Tsutsumi, K. and Ishikawa, K. (1990). Structure of chicken aldolase C mRNA and its expression in the liver and brain during development. Biochem. Int. 20, 921-929.

Saitou, N. and Nei, M. (1987). The neighbor-joining method: A new method for reconstructing phylogenetic trees. Mol. Biol. Evol. 4, 406-425.

Shih, M.-C., Lazar, G. and Goodman, H. M. (1986). Evidence in favor of the symbiotic origin of chloroplasts: Primary structure and evolution of tobacco glyceraldehyde-3-phosphate dehydrogenases. Cell 47, 73-80.

Wainright, P. O., Hinkle, G., Sogin, M. L. and Stickel, S. K. (1993). Monophyletic origin of the metazoa: An evolutionary link with fungi. Science 260, 340-342.

Wilson, A. C., Carlson, S. S. and White, T. J. (1977). Biochemical evolution. Annu. Rev. Biochem. 46, 573-639.

Zhang, R., Yatsuki, H., Kusakabe, T., Iwabe, N., Miyata, T., Imai, T., Yoshida, M. and Hori, K. (1995). Structures of cDNA encoding the muscle-type and non muscle-type isozymes of lamprey fructose bisphosphate aldolase and the evolution of aldolase genes. J. Biochem. 117, 545-553.

Zuckerkandl, E. and Pauling, L. (1965). Evolutionary divergence and convergence in proteins. In: Evolving Genes and Proteins (eds.: V. Bryson and H. J. Vogel), pp. 97-166, Academic Press, New York. 